\title{
MIXING IN CONVECTIVE THERMAL FLUXES IN UNSTEADY NON- HOMOGENEOUS FLOWS GENERATING COMPLEX THREE DIMENSIONAL VORTICITY PATTERNS.
}

\author{
J.M. Redondo ${ }^{1,2}$, J.D. Tellez ${ }^{2,3}$, M.A. Sanchez ${ }^{3}$, P. Lopez Gonzalez-Nieto ${ }^{1,3}$ \\ ${ }^{1}$ Department of Mathematics, Instituto Pluridisciplinar, Universitad Complutense de \\ Madrid, Madrid, Spain. \\ ${ }^{2}$ Department of Physics, Universitat politecnica de Catalunya, B5 Campus Nord UPCt, \\ 08034 Barcelona, Spain.(redondo@fa.upc.edu) \\ ${ }^{3}$ BEROTZA S.L., Poligono Noain-Esquirotz., 08034 Navarra, Spain \\ (berotza@berotza.org)
}

\begin{abstract}
Diffusion and scaling of the velocity and vorticity in a thermoelectric driven heating and cooling experimental device is presented in order to map the different patterns and transitions between two and three dimensional convection in an enclosure with complex driven flows. The size of the water tank is of $0.2 \times 0.2 \times 0.1 \mathrm{~m}$ and the heat sources or sinks can be regulated both in power and sign [1-3]. The thermal convective driven flows are generated by means of Peltier effects in 4 wall extended positions of $0.05 \times 0.05 \mathrm{~cm}$ each. The parameter range of convective cell array varies strongly with the Topology of the boundary conditions. Side heat and momentum fluxes are a function of Rayleigh, Peclet and Nusselt numbers, [4-6] Visualizations are performed by PIV, Particle tracking and shadowgraph. The structure of the flow is shown by setting up a convective flow generated by buoyant heat fluxes. The experiments described here investigate high Prandtl number mixing using brine and fresh water in order to form a density interface and low Prandtl number mixing with temperature gradients. The evolution of the mixing fronts are compared and the topological characteristics of the merging of the convective structures are examined for different configurations. Based on two dimensional Vorticity spectral analysis, new techniques can be very useful to determine the evolution of scales considering the multi-fractal structure of the convective flows.
\end{abstract}

Keywords: vorticity, thermoelectric convection, PIV, scaling structure, turbulence

\section{Introduction}

The spanwise and vertical structure of the vortex structures generated by a convective structure when heating or cooling an enclosed surface are obtained naturally by means of the complete Navier-Stokes equations and agree well with the experimental 2D flows. For higher Reynolds numbers and 3D turbulent flows, the vortex structure is more complex generating more fragmented structures of even smaller vortices and of vortex dipoles. We present a university-laboratory demonstration device that allows good control of transient thermoelectric driven convective models based on the control of thermal boundary conditions and flow measurements inside a closed enclosure for didactic uses. The coupling of heat transfer and electric conduction within semiconductors is important and takes into account all thermoelectric effects, including Joule and Seebeck heating, Thomson effect, Peltier cooling effect and thus allows to set up complex boundary conditions. The experimental device in order to map the different transitions between two and three dimensional convection in an enclosure and complex driven flows. The size of the water tank is of $0.2 \times 0.2 \times 0.1 \mathrm{~m}$ and the heat sources or sinks can be set up [1-3]. The convective driven flows are generated by Peltier effects in 4 wall extended positions of $0.05 \times 0.05 \mathrm{~cm}$ each. The parameter range of convective cell array varies strongly with the Topology of the boundary heat fluxes as a function of Rayleigh, Peclet and Nusselt numbers,[4-6] Visualizations are performed by PIV, Particle tracking and shadowgraph. Patterns arise by setting up a convective flow generated by a buoyant heat flux $[5,6]$.

The improvements in experimental methods and high resolution image analysis are nowadays able to detect subtle changes in the structure of the turbulence over a wide range of temporal and spatial scales, we compare the scaling shown by different mixing fronts driven by buoyancy that form convective driven 
mixing. We use PIV and density front tracking in several experimental configurations akin to geophysical overturning $[2,3]$. We parametrize the role of unstable stratification by means of the Rayleigh numbers and compare the scaling and the multifractal structure functions of the different markers used to visualize the non-homogeneous. Both reactive and passive scalar tracers are used to investigate the mixing structure and the intermittency of the flow. Different initial conditions may be compared and the mixing efficiency of the overall turbulent process also evaluated. Patterns arise by setting up a convective flow generated by a buoyant heat flux either in the base or in a side wall of the convective enclosure $[4,6]$. The experiments described here investigate high Prandtl number mixing using brine or sugar solutions and fresh water in order to form a density interface and low Prandtl number mixing with only temperature gradients [7].

The set of dimensionless parameters define conditions of numeric and small scale laboratory modeling of environmental flows. Fields of velocity, density and their gradients were computed and visualized [8, 9]. When convective heating and cooling takes place the combination of internal waves and buoyant turbulence is much more complicated if the Rayleigh and Reynolds numbers are high in order to study entrainment and mixing. The experiments described here investigate high Prandtl number mixing using salt or sugar solutions and fresh water in order to form density interfaces. The Reynolds number can be reduced adding Glicerine. The set of dimensionless parameters define different conditions of both numeric and small scale laboratory applied often in modeling environmental flows. Fields of velocity, density and their gradients are computed using advanced visualization [8, 9].

We also map the different transitions between two and three dimensional convection in an enclosure with several complex driven flows. The size of the water tank is of $0.2 \times 0.2 \times 0.1 \mathrm{~m}$ and the heat sources or sinks can be regulated both in power and sign [2-4]. The thermal convective driven flows are generated by Seebeck and Peltier effects in 4 wall extended positions of 0.05 x $0.05 \mathrm{~cm}$ each. See Figure 1 . The parameter range of convective cell array varies strongly with the Topology of the boundary conditions. At present side heat fluxes are considered and estimated as a function of Rayleigh, Peclet and Nusselt numbers, [4-8]. The evolution of the turbulent mixing layer and its complex configuration is studied taking into account the dependence on the initial modes at the early stages, Self-similar information [913]. Spectral and Fractal analysis on the images seems very useful in order to estimate dominant mixing structures as well as the basic instabilities than drive the turbulent direct and inverse cascades [12-15].

Next we describe the Thermoelectric Driven Didactive Unit presenting some experimental results obtained with PIV for selected configurations of the flow, emphasis here is just on the general capability and flow examples from an experimental point of view, more systematic pattern classification studies [1013] will be reported in due time.

\section{Laboratory equipment.}

The thermoelectric convective driven flows are generated by Peltier effect in 4 wall extended positions of $0.05 \times 0.05 \mathrm{~cm}$ each, these can be regulated independently. Another advantage of the equipment as seen in Figure 1, is the full control of the angle at which convection takes place. Because the fluid enclosure may become totally isolated, the range of zenithal and azimuthal angles span almost 180 and 360 degrees.

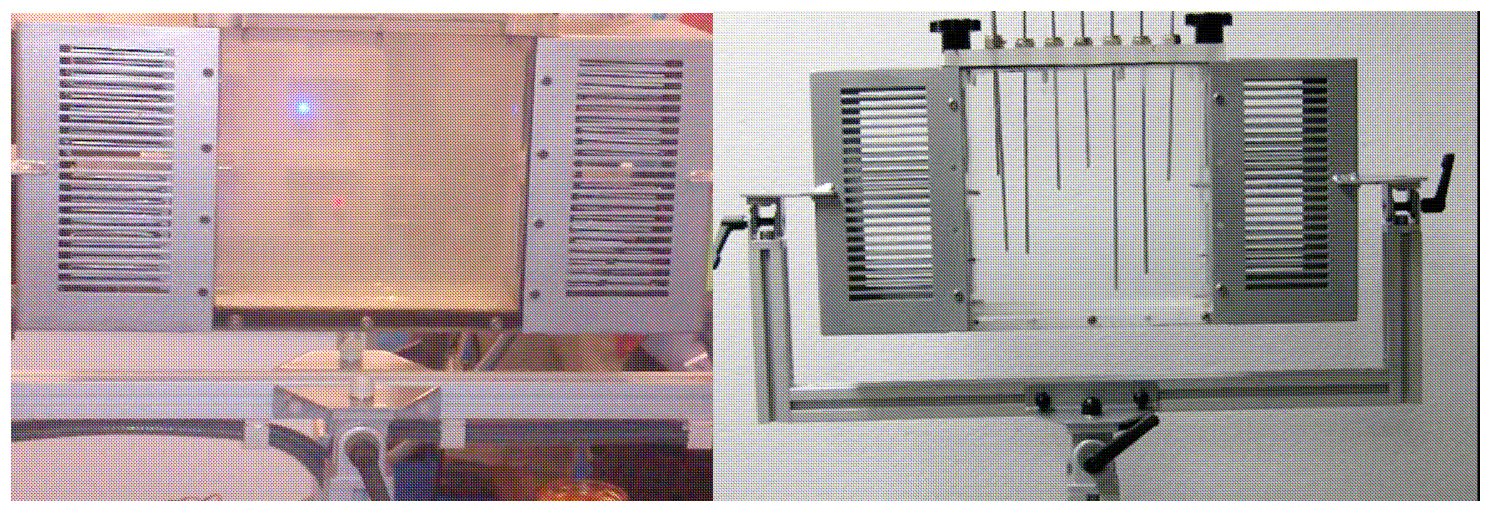

Figure 1: Details of the $\boldsymbol{T D C} \boldsymbol{U}$ experimental set up.

The basic Thermoelectric driven convective unit (TDCU) is also provided with 8 calibrated thermocouples placed near the cooling-heating side walls, the temperature measuring points may be 
registered and analyzed in any computer through the USB port with the software provided. Flow pattern visualization, Particle tracking and Particle image velocimetry are also available using PIV software. The possibilities of the $\boldsymbol{T D C} \boldsymbol{U}$ as a didactic tool fo student laboratories span the academic and technical range, from high school environmental science laboratories to research fluid dynamic laboratories. Environmental and Engineering Fluid Mechanics laboratories at university or in professional schools may incorporate student practical work in the many fields related to fluid convection that need understanding such as:

ENVIRONMENTAL FLOWS: Convection in the Atmosphere; Thermal plumes in the ABL; Sea and Mountain Breezes; Inversion layers; Thermohaline convection; Thermal and Solutal Mixing; Diffusion; Turbulence scaling

FLUID DYNAMICS AND HEAT TRANSFER: Natural Convection, Laminar and Turbulent Convection, Enclosed Flows, Bottom and Side wall Thermal Boundary layers. Rayleigh and Nusselt number evaluation. Turbulent correlation,

NUCLEAR, CIVIL AND INDUSTRIAL ENGINEERING: Ventilation, Nuclear Reactor Cooling, Thermal Stratification, Buoyant Mixing, Wall thermal correlations, Angled surfaces, Thermal Stirring and Mixing. Chemical Thermal Reactions.

The experimental apparatus placed in a standard convective takes place where heat is applied at the base and is being cooled from above. The visualization are visible when performed by "Kaliroscope" shear enhancing particles. Figures 2 and 3 show the technical details of the construction of the existing, commercially available, Thermoelectric driven convective unit. The structure and non-dimensional number parameter range of convective cell array varies strongly with the Topology of the boundary conditions and range of heat fluxes that may be applied independently to each of the four thermoelectric devices as well as the angles.

Figure 2 shows the inner mounting of the Peltier Heating/Cooling individual units attached to the walls of the Thermoelectric driven convective unit, only one side is fully expanded. In Figure 3 the details of the support with the range of angular options are drawn.

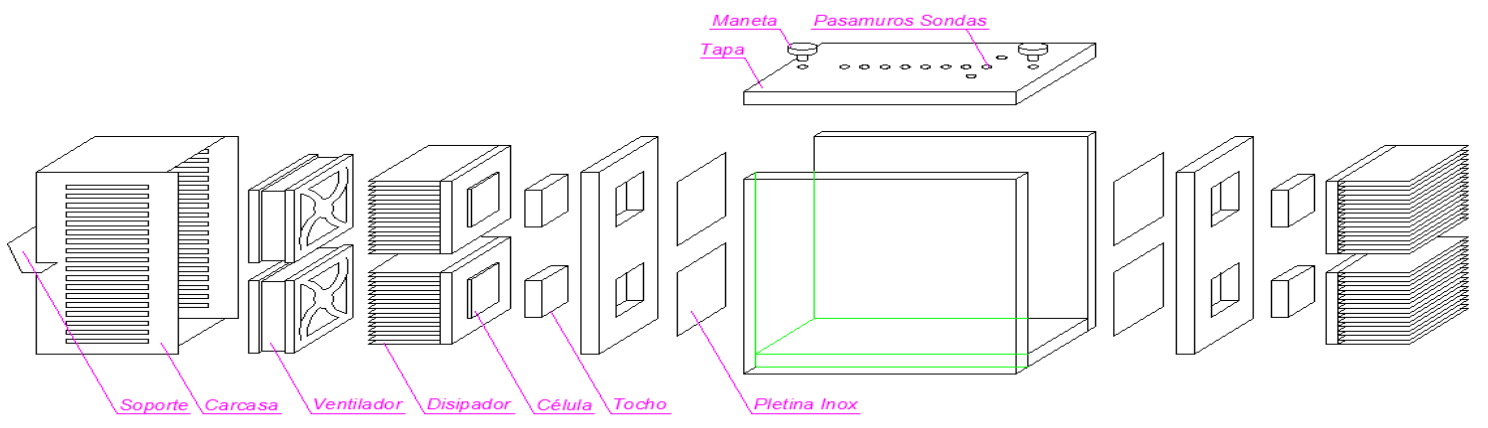

Figure 2: Technical description of the $\boldsymbol{T D C U}$, Detailed piece and technical set up, only the left side is fully described, other configurations include 8 Peltier cells.
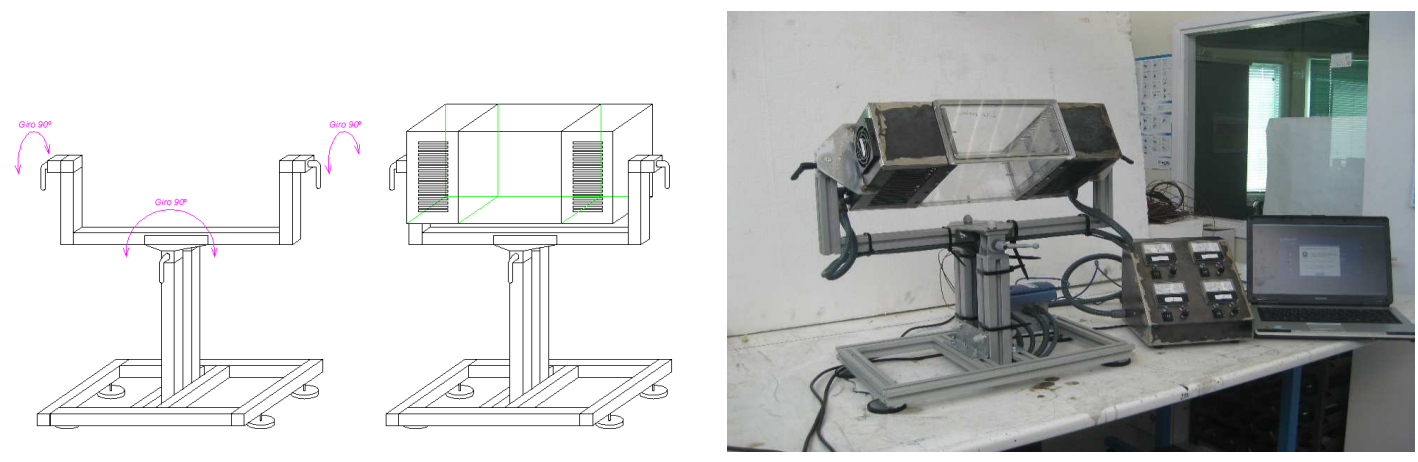

Figure 3: Positioning abilities and heating-cooling computer control

\section{Experimental Velocity Flow Results}

The design conditions were worked out for the different designed heat flows. The basic limiting working 
conditions were as follows: Minimum 20K temperature jump between hot and cold sides. Control of temperature with PDI temperature feedback in $4-8$ points to a maximum of $(0.2 \mathrm{~K})$ error. Imbalance between the heating and cooling power of $10 \%$. Flows were detected even with $0.1 \mathrm{Watts}$ of power.

Looking at the laboratory apparatus there are many different example of flows that may be set up, considering only the simplest 4 Peltier cell prototype, as shown in Figures 1-3, with two heat source-sinks in each of two opposing sides. Thanks to the possibility of rotating the sides as indicated in Figure 2, there are many different experimental configurations. Only considering the parallel or vertical angle possibilities, by orienting the visualization largest plane of $20 \mathrm{~cm} \times 20 \mathrm{~cm}$ we can either heat/cool both vertical sides or top and bottom. In each configuration, leaving aside all possible non straight angle configurations then the short list of Heating-Cooling-No Heat flux configurations, which we shall denominate as $\mathrm{H}-\mathrm{C}-\mathrm{N}$ are: $\mathrm{HHCC}, \mathrm{CCHH}, \mathrm{HCHC}, \mathrm{CHCH}$, which mean cooling at one side and heating the other side or viceverse, or Heating below at both Peltier cells and cooling above, or vv. Or other simpler velocity set ups like CCCC or $\mathrm{HHHH}$, cooling everywhere at both sides of heating at the four cells. It is also possible to set up a more complex pattern such as $\mathrm{HCCH}$ or $\mathrm{CHHC}$ meaning that a checkers type convective pattern appears.

The laboratory experiments described with this thermoelectric driven convective process will be analyzed and used to study complex mixing and to evaluate scalar or heat transport. The experiment might give some new insights on scaling thanks to the fractal or spectral methods described in [20, 21].

The method of analysis used, may be either Particle Tracking (PT) or the so called Particle Image Velocimetry (PIV), or (CIV) Correlation Image Velocimetry. In order to be able to use this method, the flow must be seeded with particles. Laser or strong parallel light is used for the side illumination of the particles and a proper detection device has to be added to the set-up [17, 18].

For the analysis of the experiment there are basically two different ways of recording the generated flow: a series of shots with a very fast photo camera or a short video with a regular camcorder. Since we had both possibilities, they were compared. From a didactic point of view, the simplest method was to us. VisualDub program to divide a short video into snapshots, then using DigiFlow or MatLab to perform $\mathrm{C} / \mathrm{PIV}$ and using ImaCalc [20] for final processing. All programs are freely available [21-25]. The option of recording the experiment was chosen for the examples presented here.

Figures 4 to 6 show the different visualization examples obtained from a convective pattern generated by heating and cooling at the side walls. In Figure 7 the enclosure has been tilted and the heating only takes place below with a configuration NHNN.

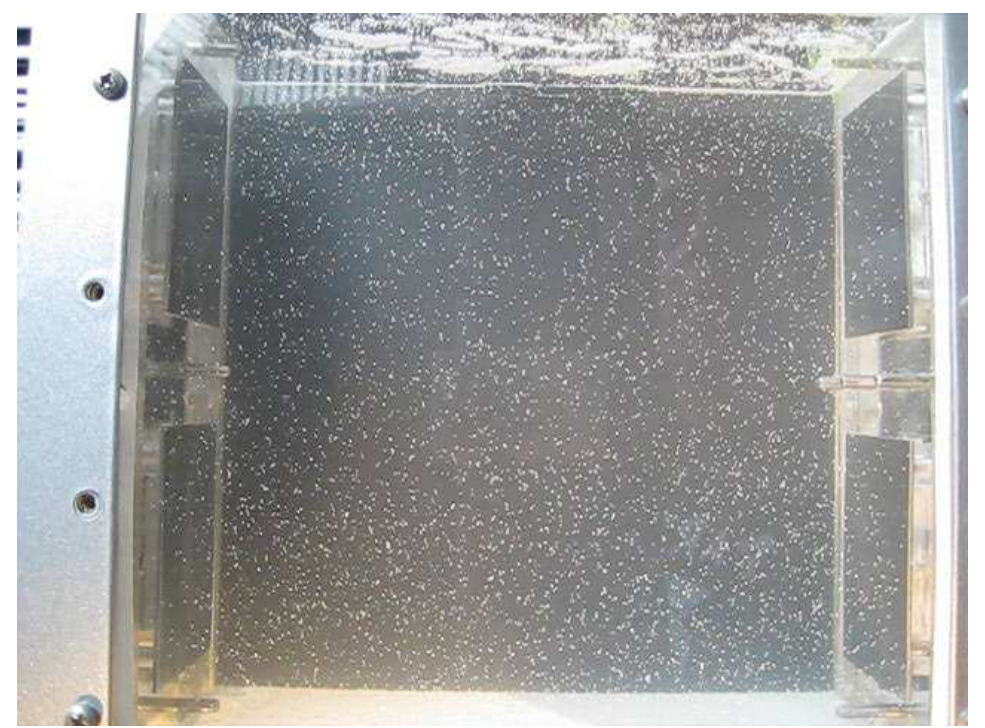

Figure 4: Enclosure with fluid (Water with Pliolite spherical-volume particles). Here Heating and cooling from the sides produces a complex convective flow shown in Figure 5. 

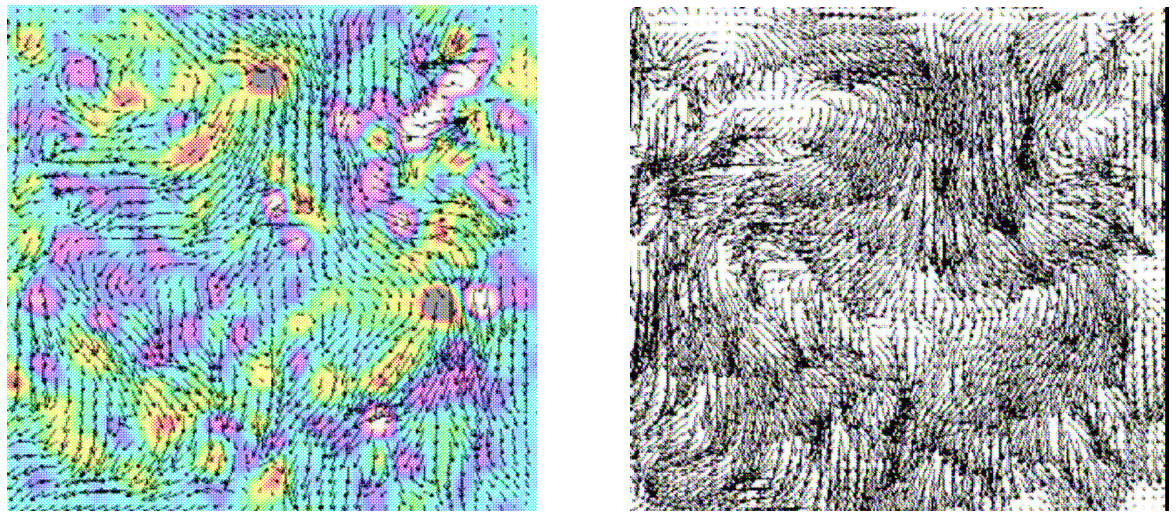

Figure 5: Flow produced by heating above and cooling below at both sides- only the central plane is presented. The configuration in 2D would be HCHC.(left) Vorticity-Velocity. (right) Higher velocity vector resolution
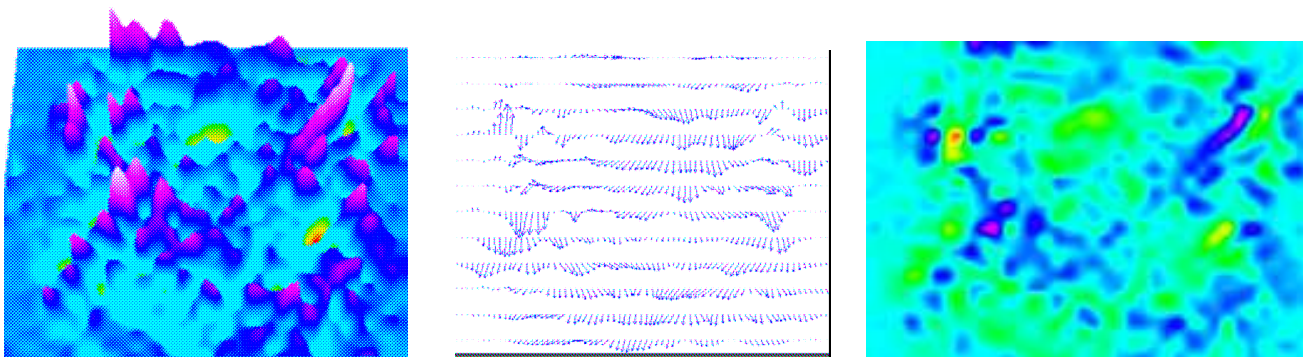

Figure 6: Details of the vorticity and velocity vertical fluxes
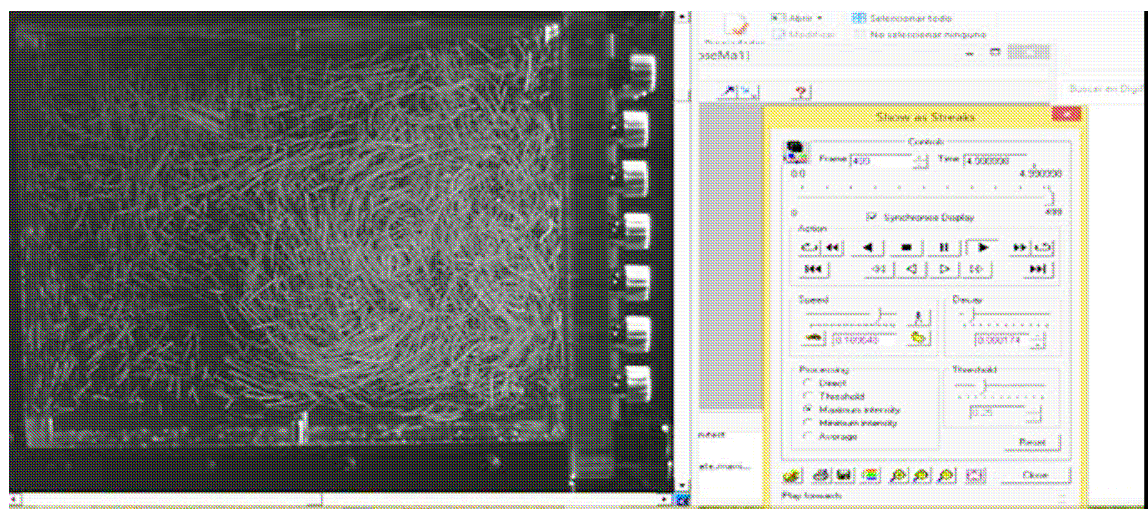

Figure 7: Particle tracks when heating from below. DigImage visualization.

Figures 9 and 10 show the instantaneous velocity and vorticity data $V(x, y, z, t)$ for a single vertical plane at the centre of the TCDD, if no 3D effects are dominant, different descriptors can be calculated per unit area of the base surface. For the convective fluid layer $r(z, t)$ may be calculated as a local $(x, y)$ vertical density (or velocity $v$ ) profile at time t. For a velocity structure function series the fractal dimension may be calculated, in which case the turbulent structure of the cascade will also be a function of the structure function of order $p$, defined as: $S(d v)^{p}=\left\{/ v(r, t)-\left.v(r-z, t)\right|^{p}\right\}$. We may then assume two types of contribution: to the velocity $v$ proceeding from active eddies generated by the local wall heat sources, but also due to the vorticity persistence and a geometrical intermittency factor, related to the number of dissipation voids that gives the volume factor occupied by eddies of vertical(buoyancy) generation with a higher intermittency exponent due to Malkus relation, with an exponent due to the experimental ( easily checked by dimensional analysis) fact that at large Reynolds number, then: $N u \sim R a^{1 / 3}$.

Figures 8-9 show the velocity and vorticity fields, when a convective single column is generated by heating at the bottom layer of the TDCU, the isolated vortices may be detected by means of a 3D image of the lateral vorticity calculated from the PIC data. 

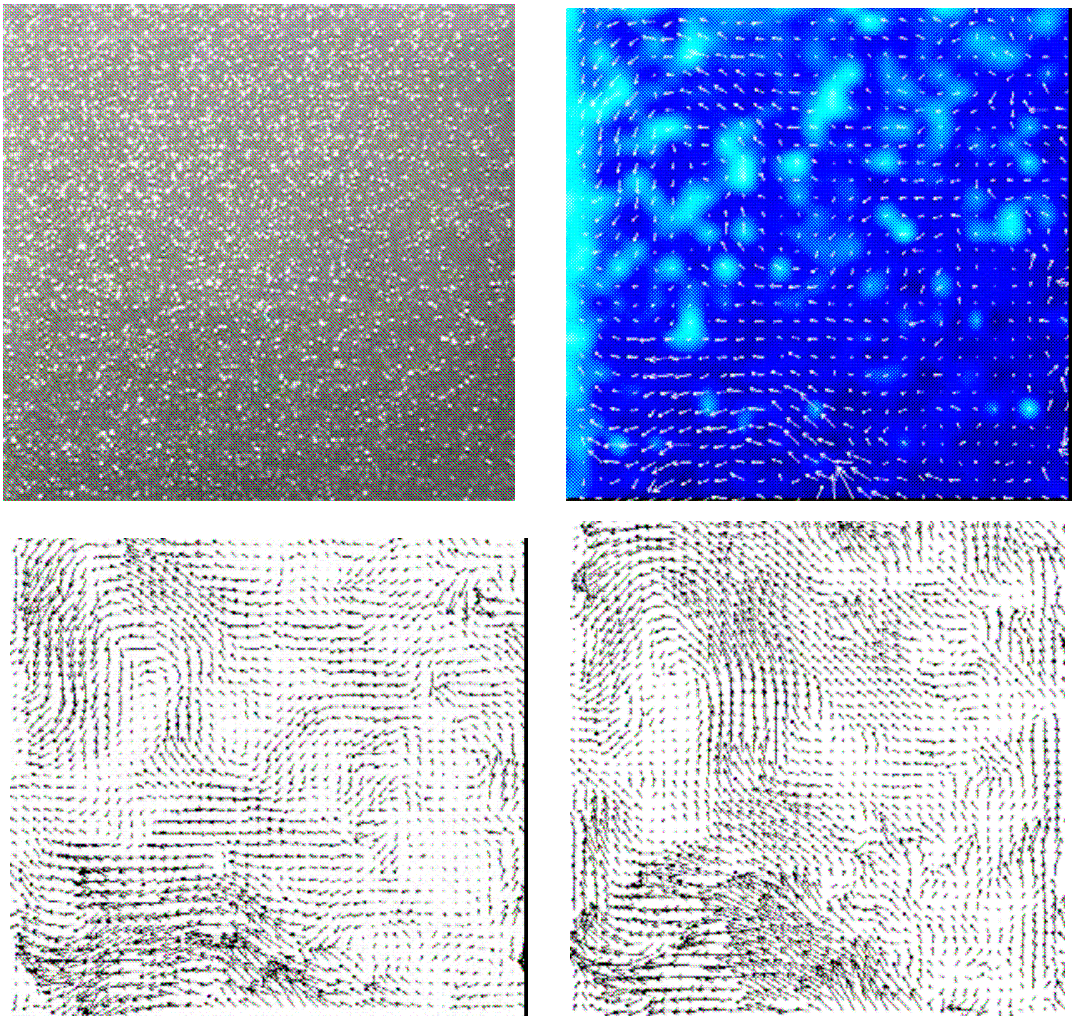

Figure 8: Heating at the base near the left wall, here the configuration could be expressed as NHNN, only heating at the bottom corner (above) Particle seeding, Correlation Velocimetry. (below)Evolution of Convective single cell.
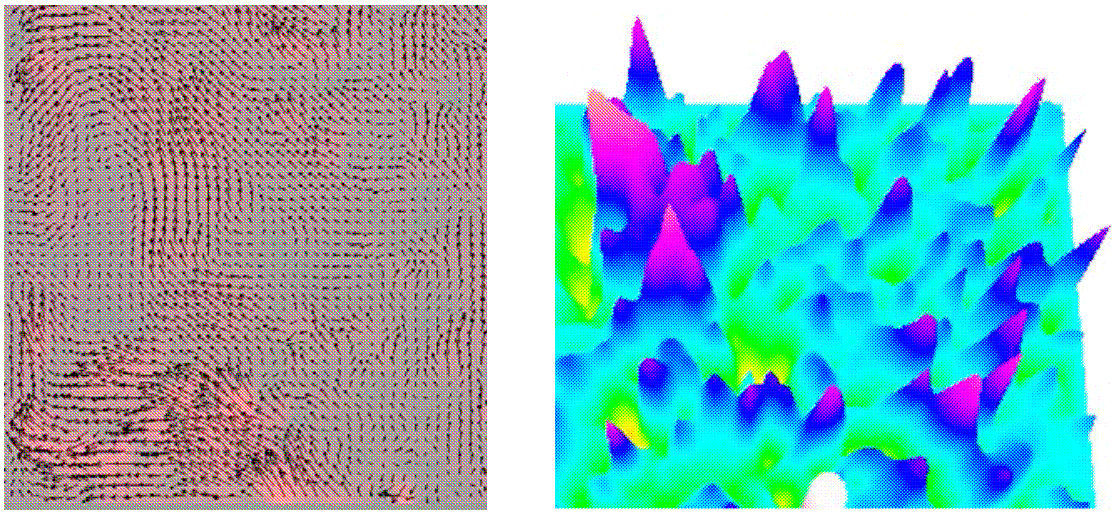

Figure 9: Vorticity distribution of the experiment shown in figure 8, with heating NHNN at a single Peltier Cell
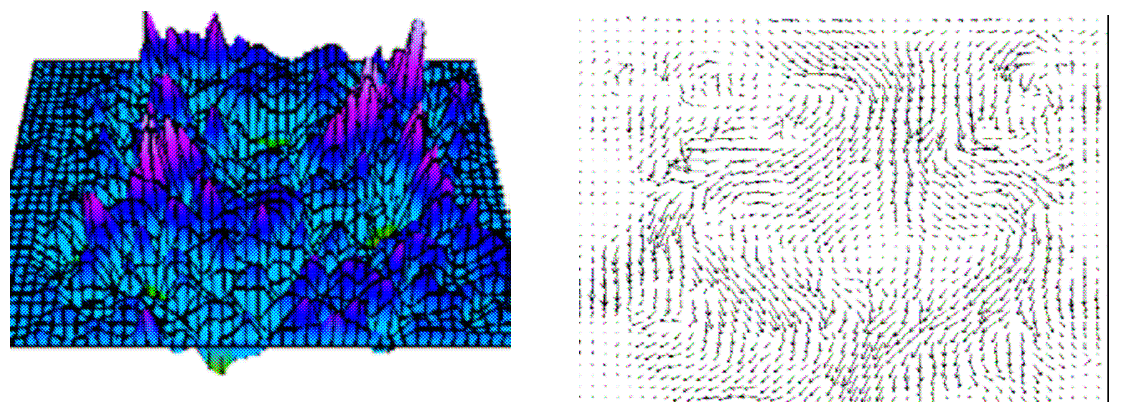

Figure 10: Structure of the convective flow HCHC from the sides 


\section{Conclusions}

This new versatile experimental apparatus allows a wide range of innovative research and practical flow visualization and analysis in fluid and turbulence laboratories [18]. An explanation for the phenomenon observed in laboratory scale models whereby the natural convection boundary layer may detach from the heated wall and form one or more horizontal intrusions into the enclosure for particular values of nondimensional heat flux area. When the heat source on the wall is switched on it is assumed that a turbulent natural convection boundary layer forms adjacent to the wall [15-18] and several instabilities may be singled out.

The growth in the boundary layer is complex. Indeed there is a great deal we do not know about the scaling of convective turbulence, with rather Bolgiano-Obukhov than Kolmogorov scaling [19,21] and the behaviour of high Rayleigh number natural convection boundary layers despite the fact that they play a very important part in the thermal behaviour of buildings, environmental, astrophysical flows and other situations. (Note: a Rayleigh number of order $10^{11}$ is typical of a large space in a building with a heated wall of height $H=5 \mathrm{~m}$ and temperature difference of $10^{\circ} \mathrm{C}$ between wall and the air). However, at high boundary layer Rayleigh number it was reasonably assumed that effects of molecular viscosity are small compared to those of turbulent mixing and that the "entrainment assumption", used frequently in the analysis of buoyant plumes and cell models held, but the effects of initial and boundary conditions seem more important than first thought $[16,23-25]$ and need a multidimensional non-dimensional parameter map.

\section{Acknowledgments}

The authors would like to thank S. Dalziel at DAMTP (Cambridge) for help and discussions on: experimental fluid dynamics. Thermoelectric devices PIV and Particle tracking with DigImage , Experiments were aided by Peter Furmanek and Teresa Vila who held Socrates-Erasmus EU fellowships. BEROTZA S.L. under CTT-UPC grant supported Jackson D. Tellez. And build the TDDU Additional support was provided by ERCOFTAC PELNoHT, SIG 14 and grant 2001SGR00221 (2009) of the Generalitat de Catalunya.

\section{References}

[1] Redondo, J.M. \& Garriga, J.: Convection driven by thermoelectric heat fluxes. in Mixing in Geophysical Flows (Eds. Redondo JM and Metais O.) CIMNE. 1995. 343-352.

[2] Redondo J.M.: Termodinámica de los procesos irreversibles, efectos termoeléctricos Rev. Termoelectricidad 2 AIT. 1992. Pamplona. pp. 16-29.

[3] Mahjoub O.B., Redondo J.M. and Babiano A.: Hierarchy flux in non-homogeneous flows. in Turbulent diffusion in the environment. Eds. Redondo J.M. and Babiano A. 2000, pp. 249-260.

[4] Redondo J.M., Sanchez J.M. Pascual I. Noriega, G.F.: Thermoelectric regulation for electric cabinets. XVI International Conference in Thermoelectrics, 02-G20. Ed. V. Vedernikov. St. Petersburg, Russia. 1995, pp. 456-468.

[5] Kuramitsu, M.; Redondo, J.M. \& Noriega, G.: Measurements of anisotropy, thermoelectric behavior and multi-fractal aspects of FeSi and of complex custom made TE materials. Twenty-Second International Conference on Thermoelectrics. 1993, pp. 541-545.

[6] López González-Nieto, P., Cano, J. L. \& Redondo, J. M.: Buoyant mixing processes generated in turbulent plume arrays. Física de la Tierra, vol. 19. 2007, pp. 205-219.

[7] Vila, T. J. Tellez, J. M. Sanchez, L. Sotillos, M. Diez \& J.M. Redondo: Diffusion in fractal wakes and convective thermoelectric flows. Geophysical Research Abstracts, Vol. 16, EGU2014-1204, 2014.

[8] Jing-Hui Meng, Xiao-Dong Wangb \& Xin-Xin: Transient modeling and dynamic characteristics of thermoelectric cooler. Applied Energy 108. 2013. 340-348.

[9] Fraunie P., Berreba S. Chashechkin Y., Velasco D. \& Redondo J.M.: LES and laboratory experiments on the decay of grid wakes in strongly stratified flows. Il Nuovo Cimento C 31, 2008. 909-930.

[10] Redondo, J.M.: The structure of density interfaces. PhD Thesis. DAMTP, University of Cambridge. 1990. 
[11] Sekula E. \& Redondo J. M.: The structure of turbulent jets, vortices and boundary layer: Laboratory and field observations, Il Nuovo Cimento, Vol. 31, N. 5, 2008. 893 - 907

[12] Platonov A., Carillo A., Matulka A., Sekula E., Grau J., Redondo J. M. \& Tarquis A. M.: Multifractal observations of eddies, oil spills and natural slicks in the ocean surface. Il Nuovo Cimento, Vol. $31 \mathrm{C}$, N. 5-6, 2009. 861-880.

[13] Redondo J.M.: Vertical microstructure and mixing in stratified flows. Advances in Turbulence VI. Eds. S. Gavrilakis et al. 1996, pp. 605-608.

[14]Redondo J.M.: Mixing efficiencies of different kinds of turbulent processes and instabilities, Applications to the environment. Turbulent mixing in geophysical flows. Eds. Linden P.F. and Redondo J.M. 2002, pp. 131-157.

[15] Tarquis, A. M., Platonov, A., Matulka, A., Grau, J., Sekula, E., Diez, M., and Redondo, J. M.: Application of multifractal analysis to the study of SAR features and oil spills on the ocean surface. Nonlin. Processes Geophys., 21, 439-450, 2014.

[16] Matulka A., Redondo J.M. \& Carrillo A.: Experiments in stratified and rotating decaying 2D flows, Il Nuovo Cimento 31, 5-6, 2008. 757-770.

[17] Castilla R., Oñate E. \& Redondo J.M.: Models, Experiments and Computations in Turbulence. CIMNE, Barcelona, 2007, pp. 255.

[18] Redondo J.M., Garriga J.N \& Noriega, G.F.: Convective Transfer in Space Thermoelectric Driven Flows. XI International Conference in Thermoelectrics, Ed. K. Rao. Arlington. Tx , 1992, 147-154.

[19] Matulka, A., López, P., Redondo, J. M. \& Tarquis, A.: On the entrainment coefficient in a forced plume: quantitative effects of source parameters, Nonlin. Processes Geophys., 21, 2014. 269-278.

[20]Dalziel, S. B.: Perturbations and coherent flow in Rayleigh-Taylor instability. 4th International Workshop on the Physics of Compressible Turbulent Mixing, ed. P. F. Linden, D. L. Youngs, \& S. B. Dalziel; 1994 32-41.

[21] Mahjoub, O.B; Redondo J.M.; Babiano A.: Structure functions in complex flows. Applied Scientific Research. 59, 299-313. 1998. Kluwer.

[22] Redondo J. M.; and Carrillo A.: Experiments in Stratified and Rotating Flows. FRAGMA. Madrid. IPD. 2010.

[23] Redondo, J.M. Tellez, J; Sotillos, L.; Gonzalez-Nieto, Pilar L.; Sanchez, J.M.; Furmanek P., Diez M.: Complex Convective Thermal Fluxes and Vorticity Structure. Geophysical Research Abstracts, Vol. 17, EGU2015-14773, 2015 - EGU General Assembly

[24] Nicolleau, F.C.G.A.; Cambon, C.; Redondo, J.M.; Vassilicos, J.C.; Reeks \& M.; Nowakowski, A.F. (Eds.): New Approaches in Modeling Multiphase Flows and Dispersion in Turbulence, Fractal Methods and Synthetic Turbulence. ERCOFTAC Series. 2012.

[25] Mahjoub O.B., Redondo J.M., and R. Alami: Turbulent Structure Functions in Geophysical Flows. Rapp. Comm. int. Mer Medit., 35, 1998, 126-127. 